\title{
Effect of potassium for cesium replacement in atomic level structure of potassium cobalt hexacyanoferrate(II)
}

\section{Puhakka, Eini}

2020-06

Puhakka , E , Ritala , M \& Lehto , J 2020 , ' Effect of potassium for cesium replacement in atomic level structure of potassium cobalt hexacyanoferrate(II) ', Radiochimica Acta, vol. 108 , no. 6 , pp. 451-457 . https://doi.org/10.1515/ract-2019-3165

http://hdl.handle.net/10138/324065

https://doi.org/10.1515/ract-2019-3165

acceptedVersion

Downloaded from Helda, University of Helsinki institutional repository.

This is an electronic reprint of the original article.

This reprint may differ from the original in pagination and typographic detail.

Please cite the original version. 


\title{
EFFECT OF POTASSIUM FOR CESIUM REPLACEMENT IN ATOMIC LEVEL STRUCTURE OF POTASSIUM COBALT HEXACYANOFERRATE(II)
}

Eini Puhakka ${ }^{1}$, Mikko Ritala $^{2}$ and Jukka Lehto ${ }^{1}$

Department of Chemistry - ${ }^{1}$ Radiochemistry, ${ }^{2}$ Materials chemistry

P.O.Box 55, FIN-00014 University of Helsinki, Finland

*Corresponding author, jukka.lehto@helsinki.fi

Keywords: potassium cobalt hexacyanoferrate; ion exchange; cesium; potassium; structure

Running title: Cs for K exchange in potassium cobalt hexacyanoferrate(II)

\begin{abstract}
Potassium cobalt hexacyanoferrate(II) $\left(\mathrm{K}_{2} \mathrm{CoFe}(\mathrm{CN})_{6}\right)$ is an extremely selective ion exchanger for cesium ions. To examine the atomic level background for the selectivity a computational structural study using DFT modelling was carried out for $\mathrm{K}_{2} \mathrm{CoFe}(\mathrm{CN})_{6}$ and for products where Cs has replaced $\mathrm{K}$ in the elemental cube cages closest to the surface. In the K-form compound the potassium ions are not in the center of the Co-Fe-CN elementary cube cages closest to the surface but locate about $140 \mathrm{pm}$ from the cube center towards the surface. When cesium ions are exchanged to these potassium ions they locate much deeper from the surface, being only about $70 \mathrm{pm}$ upwards from the cube center. This apparently leads to much stronger bonding of cesium compared to potassium. Once taken up into the outermost cube cages on the surface of the crystallites cesium ions are not able to penetrate further since they are much larger than the electron window between the cubes. Furthermore, they are not able to return to the solution phase either leading to a practically irreversible sorption.
\end{abstract}




\section{Introduction}

Potassium cobalt hexacyanoferrate(II) has been known from 1960s as an effective ion exchanger for radiocesium separations from nuclear waste effluents [1]. For the first time it was used in an industrial-scale separation process at the Loviisa nuclear power plant, Finland, in 1991 [2]. The performance in radiocesium removal was excellent: with a single 8-litre hexacyanoferrate column radiocesium could be separated from $160 \mathrm{~m}^{3}$ of waste solution containing $5 \mathrm{M}$ of potassium and the decontamination factor was very high at 2000 [3]. Since 1995, this hexacyanoferrate product has been manufactured by Fortum corporation, Finland, under the trademark CsTreat ${ }^{\circledR}$ and it has been utilized in radiocesium removal processes in a number of nuclear facilities, including Fukushima Daiichi plant [4-5].

The reason for the very high cesium selectivity of potassium cobalt hexacyanoferrate(II) has not been solved yet. This study addresses this problem by using molecular modelling techniques to explore the atomic level structure of potassium cobalt hexacyanoferrate both in the initial potassium form and when transformed into the cesium form. The structural model is based on the study of [6]. He revealed that $\mathrm{K}_{2} \mathrm{CoFe}(\mathrm{CN})_{6}$ and $\mathrm{Cs}_{2} \mathrm{CoFe}(\mathrm{CN})_{6}$ are isostructural, both having a face-centered cubic lattice. In the unit cell, there are eight elementary cubes where iron and cobalt ions are in the corners and $\mathrm{CN}$ ions are on the edges, $\mathrm{N}$ atoms facing to the Co ions and $\mathrm{C}$ atoms to the Fe ions. The exchangeable potassium ions are located inside the cubes. The unit cell parameter $a$ for the potassium form compound is $1.006 \mathrm{~nm}$ while that of the cesium form is somewhat greater at $1.032 \mathrm{~nm}$. Ceranic postulated that the great selectivity of the potassium cobalt hexacyanoferrate is due to decrease of material crystallinity and ensuing partial collapse of the structure in the cesium form leading to trapping of cesium into the cube cages. Lehto et al. [7] have shown that cesium exchange in potassium cobalt hexacyanoferrate takes place only in the outermost cube cages on the surfaces of the crystallites. Therefore, in the study reported here we focus on three types hexacyanoferrates: a product solely in the potassium form, a product where all the potassium ions closest to the surface are replaced by cesium ions, and finally a product in between with 50\%/50\% occupancy of both ions in the outermost cube cages. 


\section{Methods}

Methods based on the density functional theory (DFT) were used to investigate the crystalline and surface structures of potassium cobalt hexacyanoferrate(II). First, the structure of $\mathrm{K}_{2} \mathrm{CoFe}(\mathrm{CN})_{6}$ was optimized using literature data for the unit cell structure as background and reference information [6]. Then structures of the products where $50 \%$ and $100 \%$ of the $\mathrm{K}$ ions in the elementary cubes closest to the surface were replaced by Cs ions were solved. The calculations were performed with the CASTEP code (CAmbridge Serial Total Energy Package) by Clark, S.J. et al. [8] implemented into Materials Studio versions 8.0 [9]. The modelling is based on solving the total electronic energy and overall electronic density distribution in order to define the energetically stable structures [10]. The exchange-correlation was described with generalized gradient approximation GGA-PW91. The generalized gradient approximation GGA-PW91 was selected based on our earlier study [11] which contained same chemical elements and in order to receive comparable results. Then it was detected that GGA-PW91 gives better description of the system, and calculations achieve convergence faster than using GGA-PBE. As a compromise between the accuracy and computational time of calculations, the ultrasoft pseudopotentials were used for each element. In the potentials of cobalt and iron, the semicore states were treated as a part of the core. The used potentials were C_OOPBE.usp for carbon, Co_OOPBE.uspcc for cobalt, Cs_0OPBE.usp for cesium, Fe_0OPBE.uspcc for iron, K_0OPBE.usp for potassium, and N_OOPBE.usp for nitrogen. The kinetic cut-off energy for a plane wave expansion of the wave function was $280 \mathrm{eV}$. The morphology of potassium cobalt hexacyanoferrate was predicted by using the Bravais-Friedel Donnay-Harker (BFDH) method implemented into the Materials Studio version 8.0.

\section{Results}

Optimized unit cell structure 
In order to check the validity of the pseudopotentials and other parameters used in the calculations, the study was started be generating the unit cell structure of potassium cobalt hexacyanoferrate and then optimizing the structure (Fig. 1a and 1b). No internal water of potassium cobalt hexacyanoferrate was included in the optimization. This structure represents the bulk of the material, not the surface that will be examined in the next section. The optimized lattice parameter $a$ was somewhat higher than that presented by Ceranic [6], $1.030 \mathrm{~nm}$ versus $1.006 \mathrm{~nm}$ - this $2.4 \%$ difference is, however, well below the expected accuracy limits of the calculation method. The optimized unit cell was used to estimate the morphology of the thermodynamically most stable potassium cobalt hexacyanoferrate crystals (Fig. 1c). The crystal is terminated with the (111) and (200) planes according to the BFDH method (Fig. 2a and 2b). Out of the two crystal limiting planes (111) is very open and thus expected to undergo reconstruction. Therefore, for this study the more compact (200) plane was selected.

a)

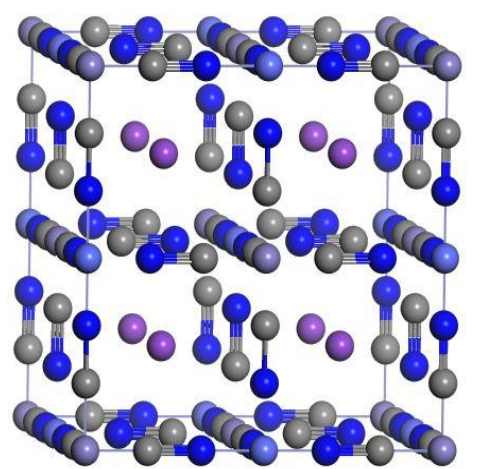

b)

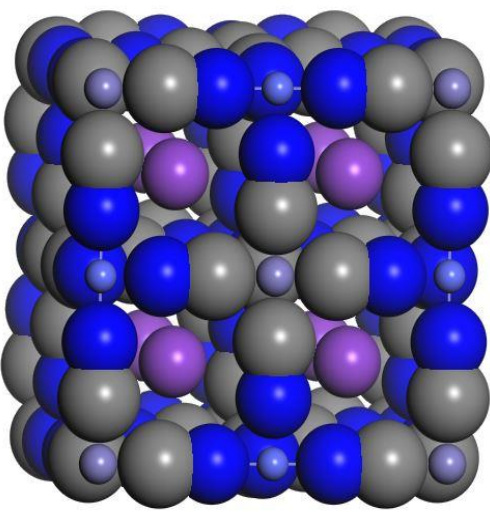

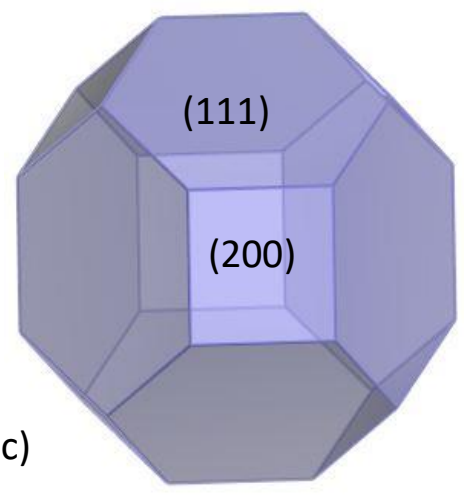

Figure 1. Potassium cobalt hexacyanoferrate(II). a) The optimized unit cell. b) The optimized unit cell with vdW spheres. c) BFDH predicted morphology. Grey spheres: carbon. Big blue spheres: nitrogen. Small blue spheres: cobalt. Small greyish blue: iron. Big lilac spheres: potassium. 
a)

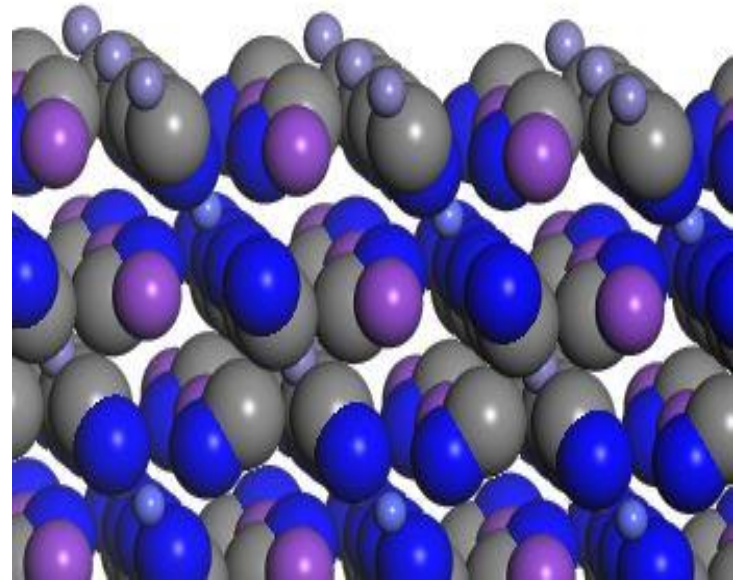

b)

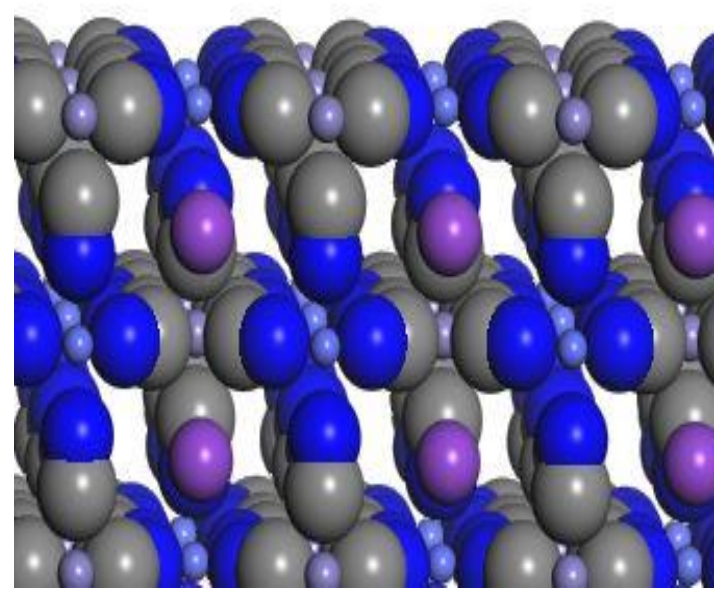

Figure 2. Crystal faces of potassium cobalt hexacyanoferrate based on the BFDH method. a) The (111) crystal face, and b) the (200) crystal face.

Effect of potassium for cesium replacement on the structure

Figures 3 and 4 present the structure of potassium cobalt hexacyanoferrate in three forms: the product fully in the K-form (upper picture), the cube cages closest to the crystallite surfaces $50 \% / 50 \%$ in the $\mathrm{K}$ - and Cs-forms (middle picture), and the cube cages closest to the surface $100 \%$ in the Cs-form (lower picture). The energetically most stable structure is the K-form, which is 5.3 $\mathrm{eV}$ or $6.0 \mathrm{eV}$ more stable than the $\mathrm{K}-50 \% / \mathrm{Cs}-50 \%$ form and Cs-form, respectively. This indicates that the structures containing the $\mathrm{Cs}^{+}$ions are more flexible than the pure $\mathrm{K}$-form. Figures 3 and 4 and Table 1 show that compared to the bulk of the K-form compound (third cube layer from the surface) the outermost cube layer has expanded in thickness by $2.4 \%$ (12 pm) while the cube layer between the bulk and the outermost cube layers has shrunk by $3.1 \%(15 \mathrm{pm})$. The middle cube layer has a thickness of $493 \mathrm{pm}$ independent of the ionic form of the outermost layer while the thickness of the outermost cube layer is $521 \mathrm{pm}$ in the K-form and a slightly less at $515 \mathrm{pm}$ both in the mixed K/Cs-form and Cs-form. Thus, exchange of potassium ions by cesium ions leads to a contraction of the outermost cube layer by $6 \mathrm{pm}$. It is important to note that the dimensions of all the cubes, 493 - 521 pm, are considerably larger than the diameters of potassium and cesium ions (ionic diameter 274 pm and 334 pm, respectively, for six-coordination) and thus the 
coordination environment inside the cubes is not optimal. Furthermore, visual inspection of Figure 3 reveals that potassium has moved towards the surface in the outer layers, and more so in the outermost layer than in the second layer. This shift is shown in a more detailed way in Figure 4: in the outermost cube layer, the distance of potassium ions from the outermost atomic layer of the cube is only $122 \mathrm{pm}$ compared to $254 \mathrm{pm}$ in the bulk. The electron density of the outermost plane is higher than in the bulk. This is probably because of the discontinuity of the crystal structure on the surface, i.e. the lack electron attractive $\mathrm{K}^{+}$ions outside the atomic plane. Also, because of the absence of potassium ions outside this plane the potassium ions in the first cubes do not feel repelling force from the outside direction unlike potassium ions in the bulk, which thus drives them towards the surface in the first cubes. Furthermore, as already mentioned, the coordination of potassium ions in the cube is not optimal due to the large size of the cube compared to the size of the potassium ion. These factors probably are the reason for the occurrence of potassium ions close to the surface instead of being in the center of the cube.

When exchanged to the outermost potassium ions, the cesium ions intruse deeper into structure than the potassium ions. This is indicated by the fact that the distance of cesium ions from the outermost atom layer is clearly longer than the distance of potassium ions in the K-form: $192 \mathrm{pm}$ in the half-exchanged form and $195 \mathrm{pm}$ in the fully exchanged form. In other words, cesium ions adopt $70 \mathrm{pm}$ and $73 \mathrm{pm}$ deeper positions than potassium ions. Interestingly, in the 50\%/50\% form the cesium and potassium ions occupy nearly the same positions as in the full cesium and potassium forms, that is, clearly different from each other. It is understandable that the center of cesium ions is deeper in the cube since it has a larger size than potassium ion. This difference explains, however, only about $30 \mathrm{pm}$ compared to the observed about $70 \mathrm{pm}$ difference. It is probable that cesium ions as larger ions than potassium ions have interaction with the negative $\mathrm{CN}$ ligands also at the bottom plane of the first cube layer, thus driving them deeper into the cage. Furthermore, Fig. 4 shows that the electron density of the surface increases when going from the $\mathrm{K}$-form to the Cs-form. When all the $\mathrm{K}$ ions are exchanged to the $\mathrm{Cs}$ ions in the outermost cube cages, the surface charge density is more evenly spread than in the full K-form 
and the $\mathrm{K} 50 \% / \mathrm{Cs} 50 \%$-form. This finding together with the structural changes may explain why the $\mathrm{Cs}$ ions are not able to return to the solution phase.
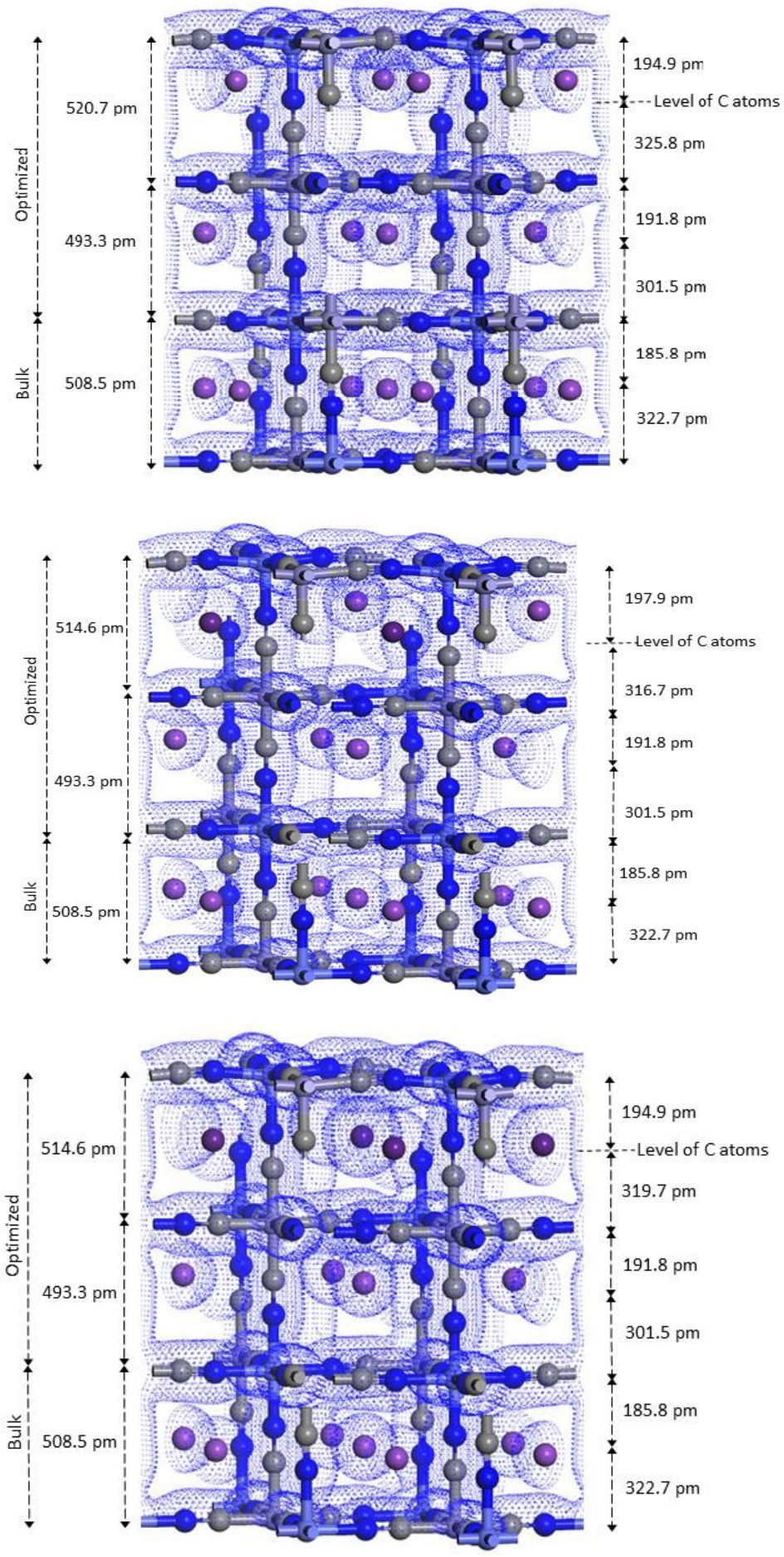
Figure 3. First three cube layers of $\mathrm{K}_{2} \mathrm{CoFe}(\mathrm{CN})_{6}$ fully in the $\mathrm{K}$-form (upper picture), the cube cages closest to the surface of $\mathrm{K}_{2} \mathrm{CoFe}(\mathrm{CN})_{6}$ crystallites are $50 \% / 50 \%$ in the $\mathrm{K}$ - and $\mathrm{Cs}$-forms (middle picture) and the cube cages closest to the surface of $\mathrm{K}_{2} \mathrm{CoFe}(\mathrm{CN})_{6}$ crystallites are $100 \%$ in the Cs-form (lower picture).

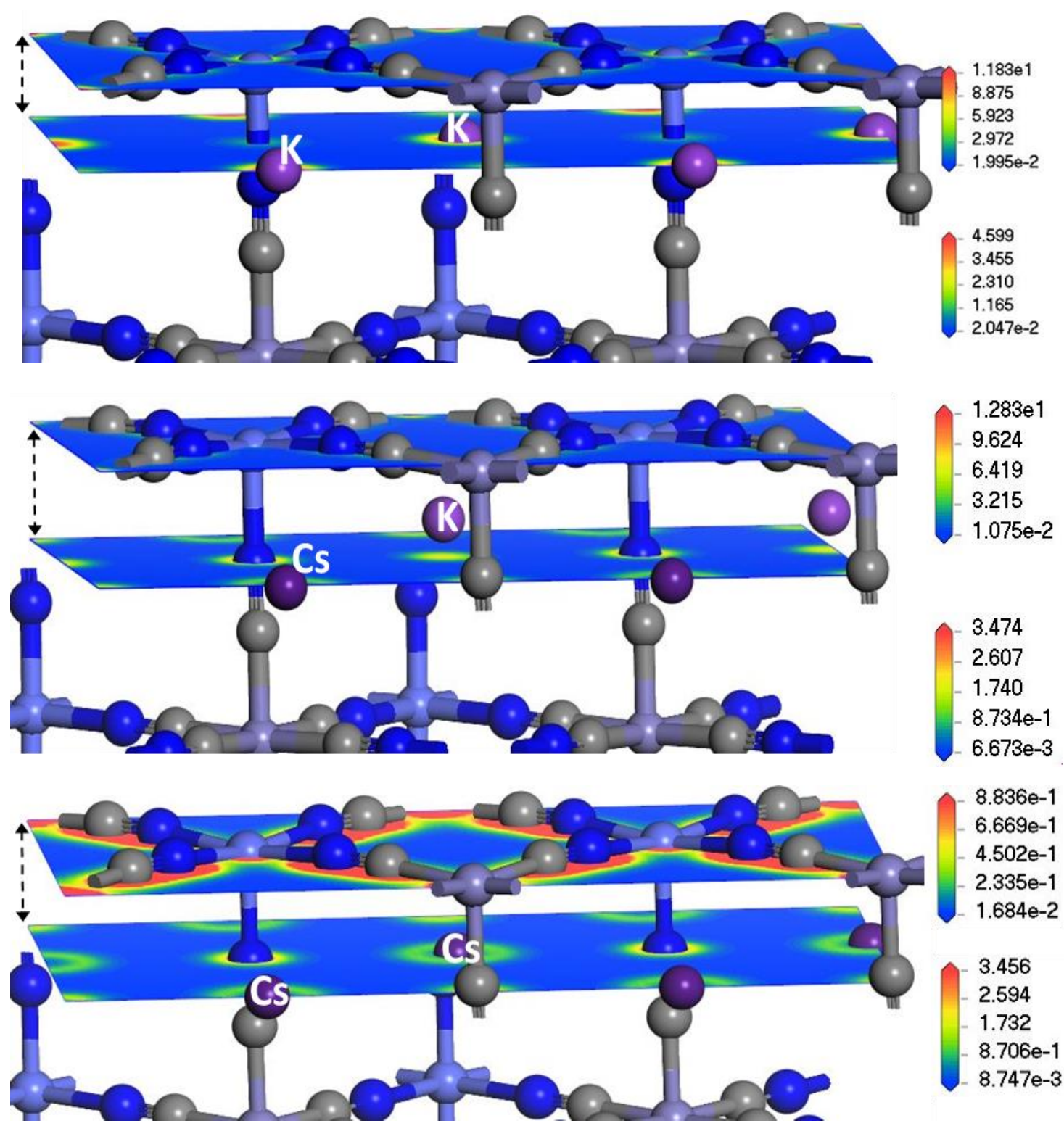

Figure 4. $\mathrm{K}_{2} \mathrm{CoFe}(\mathrm{CN})_{6}$ fully in the $\mathrm{K}$-form (upper picture), the cube cages closest to the surface of $\mathrm{K}_{2} \mathrm{CoFe}(\mathrm{CN})_{6}$ crystallites are $50 \% / 50 \%$ in the $\mathrm{K}$ - and Cs-forms (middle picture) and the cube cages closest to the surface are $100 \%$ in the Cs-form (lower picture). The distances 
between two selected charge density layers containing the surface ions and either $\mathrm{K}$ or Cs ions are 122 pm, 192 pm and 195 pm for the K-form, K-50\%/Cs-50\%-form and Csform, respectively. The scales on the right are electron densities (electrons $/ \AA^{3}$ ).

Table 1. Structural changes of potassium cobalt hexacyanoferrates according to the K/Cs-ion content.

\begin{tabular}{|c|c|c|c|c|c|c|}
\hline \multirow{2}{*}{$\begin{array}{l}\text { Changes in cubic } \\
\text { layers }\end{array}$} & \multicolumn{2}{|c|}{ K-form } & \multicolumn{2}{|c|}{ K-50\%/Cs-50\% } & \multicolumn{2}{|c|}{ Cs-form } \\
\hline & $\%$ & $\mathrm{pm}$ & $\%$ & $\mathrm{pm}$ & $\%$ & $\mathrm{pm}$ \\
\hline Outermost & +2.4 & 12.2 & +1.2 & 6.1 & +1.2 & 6.1 \\
\hline Middle & -3.1 & -15.2 & -3.1 & -15.2 & -3.1 & -15.2 \\
\hline \multicolumn{7}{|c|}{ Movement of ions in the outermost cube layer } \\
\hline $\mathrm{K}^{+}$ & +52.1 & 135.5 & & & & \\
\hline $\mathrm{Cs}^{+}$ & & & +24.6 & 62.5 & +23.4 & 59.4 \\
\hline
\end{tabular}

The window in the electron cloud between the elementary cubes can be approximated to a quadrat/quadrangle (Figure 5). In the bulk the edge of the electron cloud window can be estimated to $265 \mathrm{pm}$ and on the surface to $266 \mathrm{pm}$. In the Cs-exchanged forms, the dimensions of the quadrangle are slightly smaller than those of the K-form quadrat. The ionic diameter of sixcoordinated potassium ion $\mathrm{K}^{+}$, the probable form in aqueous solution phase, is $274 \mathrm{pm}$, approximately the same as the largest dimension of the window. Within the outermost cubes the coordination number of potassium ion is probably 8 since it is close to the surface Fe-CN-Co atomic plane and thus probably has no (or weak) interaction with the second Fe-CN-Co atomic plane. The coordination environment is not symmetrical and thus not ideal. The diameter of sixcoordinated cesium ion $\mathrm{Cs}^{+}$is considerably larger than potassium at $334 \mathrm{pm}$. Thus, in order to allow cesium ion to enter the cube cage the electron cloud needs to deform/stretch during the replacement process. After entering the cage cesium ion experiences a different ligand environment than in the solution phase. Assuming all the $\mathrm{CN}$ ions as ligands the coordination number of cesium ion within the cage is 12 for which the ionic size is greater at $376 \mathrm{pm}$ in 
diameter. Coordination number 8 in the uppermost cube is also possible since the cesium ions are not in the centre of the cubes: we see from Figure 3 that the electron cloud of cesium ion does not interact with the $\mathrm{CN}$ ions in the second Fe-CN-Co atomic plane. For the coordination number 8 the ionic diameter of cesium is $348 \mathrm{pm}$ - still too large to exit the cube. Figure 3 shows visually the difference between the potassium and cesium ions: the latter take larger space within the cubes than the potassium ions and interact with the surrounding ligands in a stronger manner. This can also be seen in Figure 6 where the uppermost cube of the (200) surface of potassium cobalt hexacyanoferrate is shown for the fully K-form and Cs-form. Furthermore, and most importantly with respect to the high selectivity, the size of the cesium ion is too large to move further into the crystallite and also too large to return to the solution phase. This explains the observed fact that cesium ions are taken up by potassium cobalt hexacyanoferrate in a practically irreversible manner [12].

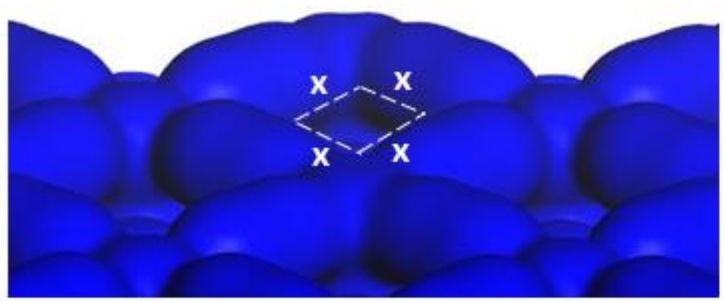

$$
x=265.0 \mathrm{pm}
$$

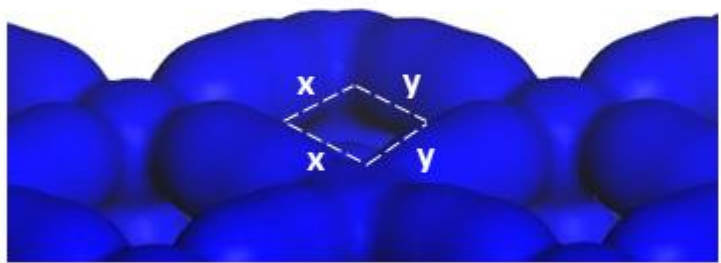

$\mathrm{x}=265.7 \mathrm{pm}$

$\mathrm{y}=265.9 \mathrm{pm}$

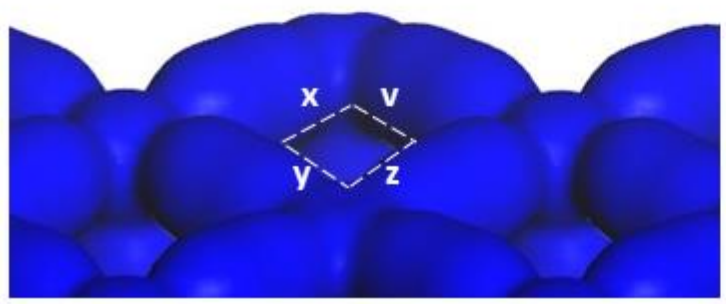

$$
\begin{aligned}
& x=265.1 \mathrm{pm} \\
& y=265.0 \mathrm{pm} \\
& z=265.9 \mathrm{pm} \\
& v=265.0 \mathrm{pm}
\end{aligned}
$$

Figure 5. Windows of the electron clouds on the surface of potassium cobalt hexacyanoferrate(II) in the K-form (upper), mixed K/Cs-form (middle) and Cs-form (bottom). 
a)

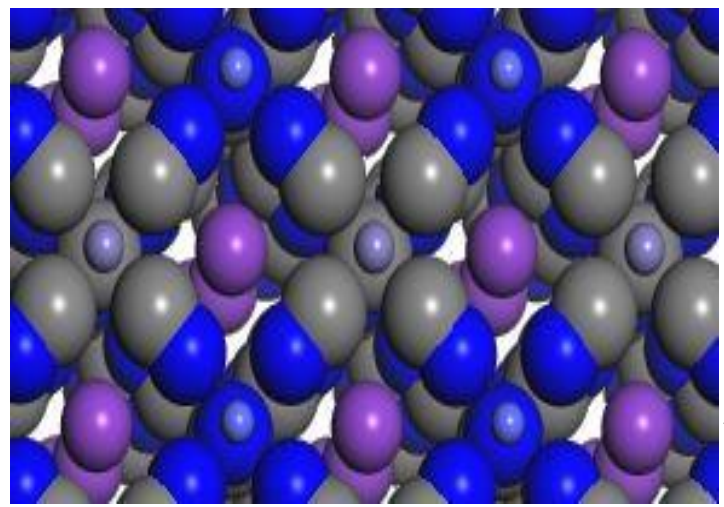

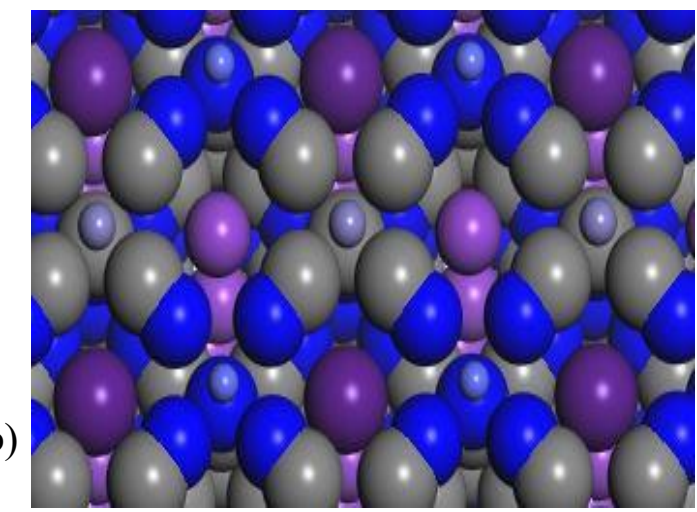

Figure 6. A top view of the (200) surface of $\mathrm{K}_{2} \mathrm{CoFe}(\mathrm{CN})_{6}$ a) fully in the K-form, and b) in Cs-form.

\section{Conclusions}

Exchange of potassium for cesium in potassium cobalt hexcyanoferrate (II) leads to practically irreversible sorption in the cube cages closest to the surface of the crystallites. The reason to this is that cesium ions are located about $70 \mathrm{pm}$ deeper in the structure than potassium ions. The coordination environment of cesium ions alters when they enter the cube cages. In the solution phase the most probable coordination number of cesium is 6 while in the cube cages closest to the surface it is 8 or 12 . This increases the size of cesium ions preventing their transfer deeper into crystallites and, most importantly with respect to the selectivity, prevents their return into the solution.

\section{References}

1. Prout, W.E., Russel. E.R. and Groh, H.J.: Ion exchange adsorption of cesium by potassium hexacyanocobalt(II)ferrate, Journal of Inorganic and Nuclear Chemistry 27, 473 (1965). 
2. Harjula, R., Lehto, J., Tusa, E. and Paavola, A.: Industrial Scale Removal of Cesium with Hexayanoferrate Exchangers - Process Development, Nuclear Technology 107, 272 (1994).

3. Tusa, E., Paavola, A., Harjula, R. and Lehto, J.: Industrial scale removal of cesium with hexayanoferrate exchangers - Process realization and test run, Nuclear Technology 107, 279 (1994).

4. Harjula, R., Lehto, J., Paajanen, A., Brodkin, L. and Tusa, E.: Removal of Radioactive Cesium from Nuclear Waste Solutions with a Transition Metal Hexacyanoferrate Ion Exchanger CsTreat $^{\circledR}$, Nuclear Science and Engineering 137, 206 (2001).

5. Lehto, J., Koivula, R., Leinonen, H., Tusa, E. and Harjula, R.: Removal of Radionuclides from Fukushima Daiichi Waste Effluents, Separation \& Purification Reviews 48, 122 (2019).

6. Ceranic, T.: The structure of an inorganic ion exchanger cobalt(II)-hexacyanoferrate(II), Zeitschrift für Naturforschung 33b, 1484 (1978).

7. Lehto, J., Haukka, S., Harjula, R., and Blomberg, M.: Mechanism of Caesium Ion Exchange on Potassium Cobalt Hexacyanoferrates(II), Journal of the Chemical Society, Dalton Transactions 3, 1007 (1990).

8. Clark, S.J., Segall, M.D., Pickard, C.J., Hasnip, P.J., Probert, M.J., Refson, K. and Payne, M.C., First principles methods using CASTEP, Zeitschrift für Kristallographie, 220, 567-570 (2005).

9. Dassault Systèmes, Materials Studio, Release 8.0. San Diego: Dassault Systèmes BIOVIA Corp., 2014.

10. Leach, A.R., Molecular Modelling, Principles and Applications, 2nd ed., Pearson Education Limited, Essex, 2001.

11. Seppälä, A., Puhakka, E., and Olin, M., Effect of layer charge on the crystalline swelling of $\mathrm{Na}^{+}, \mathrm{K}^{+}$and $\mathrm{Ca}^{2+}$ montmorillonites: DFT and molecular dynamics studies. Clay Minerals, 51, 197-211 (2016).

12. Lehto, J., Harjula, R., Haukka, S., and Wallace, J. Solidification of ${ }^{137}$ Cs into potassium cobalt hexacyanoferrate(II) ion exchanger. Proceedings of the 1989 Joint International Waste Management Conference, Kioto, Japan, October 22-28, 1989, Vol. 1, p. 367. 